\title{
C-terminus of OX2R significantly affects downstream signaling pathways
}

\author{
CHUNMEI WANG ${ }^{1}$, CHAO XU $^{1}$, MINGHUI LIU ${ }^{1}$, YANYOU PAN ${ }^{1}$, BO BAI $^{1}$ and JING CHEN $^{1,2}$ \\ ${ }^{1}$ Neurobiology Institute, Jining Medical University, Jining, Shandong 272067, P.R. China; \\ ${ }^{2}$ Division of Biomedical Sciences, Warwick Medical School, University of Warwick, Coventry CV4 7AL, UK
}

Received November 3, 2016; Accepted March 30, 2017

DOI: $10.3892 / \mathrm{mmr} .2017 .6557$

\begin{abstract}
The human orexin 2 receptor (OX2R) is a G-protein-coupled receptor (GPCR) that has been implicated in a number of diverse physiological functions. Recent studies have identified a number of functions of the $\mathrm{C}$-termini of GPCRs. However, the importance of the OX2R C-terminus in regulating signaling and surface expression remains unclear. In the present study, the function of the OX2R C-terminus was investigated using three $\mathrm{C}$-terminal mutants, which were truncated at residues 368, 384 and 414, respectively, and the wild-type control, which expressed the full-length OX2R. HEK-293 cells were transfected with the mutated and control OX2R constructs. ELISA, western blot analysis and calcium assays were used to investigate the effects of the mutations on OX2R function. The present results demonstrated that residues 385-414 and 415-444 exhibited a cumulative effect on the surface expression of OX2R. Residues 369-384 exhibited a significant influence on inositol phosphate production and extracellular signal-regulated kinase $1 / 2$ phosphorylation. Residues 385-414 significantly influenced agonist-induced internalization, whereas residues 369-384 and 385-414 significantly influenced $\mathrm{Ca}^{2+}$ release. The results of the present study suggest that the $\mathrm{C}$-terminus of $\mathrm{OX} 2 \mathrm{R}$ is important for its role
\end{abstract}

Correspondence to: Professor Bo Bai, Neurobiology Institute, Jining Medical University, 16 Hehua Road, Jining, Shandong 272067, P.R. China

E-mail: baibo1@mail.jnmc.edu.cn

Dr Jing Chen, Division of Biomedical Sciences, Warwick Medical School, University of Warwick, Medical School Building, Gibbet Hill Road, Coventry CV4 7AL, UK

E-mail: jing.chen@warwick.ac.uk

Abbreviations: aa, amino acids; ELISA, enzyme-linked immunosorbent assay; ERK1/2, extracellular signal-regulated kinase 1/2; GnRH-R, gonadotropin-releasing hormone receptor; GPCR, G-protein-coupled receptor; HA, hemagglutinin; IBMX, 3-isobutyl-1-methylxanthine; IP, inositol phosphate; OX2R, orexin 2 receptor

Key words: orexin 2 receptor, C-terminus, cell surface expression, internalization, signal transduction in various physiological and pathological processes, and may therefore be associated with such disorders as depression and anorexia.

\section{Introduction}

G-protein-coupled receptors (GPCRs) constitute one of the largest receptor superfamilies. By binding to different ligands, GPCRs activate different $G$ subunits, which subsequently induce different intracellular signaling pathways and lead to a number of diverse biological effects $(1,2)$. In general, GPCRs contain an extracellular amino terminus, a seven-transmembrane helix, and an intracellular C-terminus (3). The extracellular and transmembrane regions are primarily involved in ligand recognition, while the cytoplasmic region participates in the interaction with G-proteins or additional proteins (4). The C-termini of GPCRs differ in terms of their length and structure, implying their functional importance and complexity (5). The C-terminus is indispensable for the functions of GPCRs, including cell surface localization (6), G-protein coupling (7), agonist-driven internalization (8) and signal transduction (9). Therefore, mutations in the C-termini of GPCRs have been associated with a variety of dysfunctions and disease states $(10,11)$.

The human orexin 2 receptor (OX2R), also known as the hypocretin receptor, is a GPCR and exhibits an equal binding affinity for its two ligands, orexin-A and orexin-B (12). OX2R is involved in a number of diverse physiological actions, including control of feeding and energy homeostasis (13), control of the sleep-wake cycle (14), regulation of cardiovascular functions (15), and the neuroendocrine system (16). Thus, OX2R is implicated as a potential therapeutic target in various disorders associated with OX2R mutations. The mRNA sequence of OX2R contains 1,843 bases that are translated into 444 amino acids (aa), and its C-terminus contains 78 residues. OX2R may interact with multiple G-proteins $\left(G_{s}, G_{q}\right.$ and $\left.G_{i}\right)$ and stimulate cyclic adenosine monophosphate (cAMP) and inositol trisphosphate production (17). Despite its functional implications, no comprehensive studies have been conducted to date to determine the role of the $\mathrm{C}$-terminus in governing the surface expression and signaling of OX2R.

Basic experiments to identify functional domains in the C-termini of GPCRs usually focus on truncations of the 
C-terminus. Therefore, in the present study, $3 \mathrm{C}$-terminal truncation mutants of OX2R were employed to determine which segments are involved in its cell surface expression and signal transduction. The three mutants were truncated at the 368 (HA-M1), the 384 (HA-M2), and the 414 aa positions (HA-M3), respectively (Fig. 1A). The effects of these mutations on the normal function of OX2R were subsequently evaluated. The results may enhance the current understanding of the OX2R complex, and provide a theoretical basis for elucidating the molecular mechanisms underlying OX2R-associated diseases.

\section{Materials and methods}

Materials. Orexin-A and orexin-B were purchased from Phoenix Pharmaceuticals (St. Joseph, MO, USA). Lipofectamine 2000 and geneticin (G418) were obtained from Invitrogen; Thermo Fisher Scientific, Inc. (Waltham, MA, USA). Forskolin and 3-isobutyl-1-methylxanthine (IBMX) were obtained from Sigma-Aldrich; Merck Millipore (Darmstadt, Germany). Primary antibodies against hemagglutinin (HA; cat no. 3724), phosphorylated (p)-extracellular signal-regulated kinase 1/2 (ERK1/2; cat no. 9101), and ERK1/2 (cat no. 4696) were purchased from Cell Signaling Technology, Inc. (Danvers, MA, USA). Anti- $\beta$-actin primary antibody (cat no. TA-09), polyclonal goat anti-rabbit (cat no. ZB-2301) or anti-mouse secondary antibodies (cat no. ZB-2305) were obtained from ZSGB-BIO (Beijing, China).

Generation of expression constructs. The following 3 truncated OX2R C-terminal region constructs were generated: M1, terminating at Ser368 to remove the intracellular C-terminal tail; M2, terminating at Leu384 to leave a truncated intracellular C-terminal tail; M3, terminating at Phe414 to leave an intracellular C-terminal tail. The oligonucleotides 5'-CCCAAGCTTATGTCCGGCACCAAATTG GAGG-3' and 5'-CGCGGATCCCTATTTTCCACTGAGA AAATTATAAAT-3' introduced a stop codon following serine 368. The oligonucleotide 5'-CGCGGATCCCTATCC AAGGCAACAGCAAGAAAACGC-3' introduced a stop codon following Leu384. The oligonucleotide 5'-CGCGGA TCCCTAATCAAAGTTGCTGATTTGAGTGGT-3' introduced a stop codon following Phe414. The full-length human OX2R gene was cloned in the pcDNA3.1 vector and was used as a template. The amplified gene fragments were cloned into the HindIII and BamHI restriction sites of the pcDNA3.1 (+) vector. All constructs were confirmed by DNA sequencing using the DNA Analyzer ABI 3730xl (Applied Biosystems; Thermo Fisher Scientific, Inc.). The following primer sequences were used: $\mathrm{T} 7$ promoter, 5'-TAATACGACTCACTATAGGG-3'; and bovine growth hormone reverse primer, 5'-TAGAAGGCACAGTCGAGG-3'.

To generate HA-tagged OX2R fragments, the sequence encoding the HA epitope tag (YPYDVPDYA) was inserted into the pcDNA3.1-OX2R and the 3 truncated C-terminal $\mathrm{OX} 2 \mathrm{R}$ constructs at the $\mathrm{N}$-terminus by polymerase chain reaction. The resulting constructs (HA-OX2R, HA-M1, HA-M2, and HA-M3) were confirmed by sequencing by Sangon Biotech Co., Ltd. (Shanghai, China).
Cell culture and transfection. HEK293 cells were cultured in minimum essential medium (Gibco; Thermo Fisher Scientific, Inc.) containing 10\% fetal calf serum (Gibco; Thermo Fisher Scientific, Inc.) at $37^{\circ} \mathrm{C}$ in a $5 \% \mathrm{CO}_{2}$ incubator. To generate cell lines stably expressing HA-OX2R, HA-M1, HA-M2, and HA-M3, HEK293 cells were transfected with the plasmid constructs using Lipofectamine ${ }^{\circledR} 2000$ according to the manufacturer's protocol (Invitrogen; Thermo Fisher Scientific, Inc.). At $48 \mathrm{~h}$ following transfection, the culture media was removed and replaced with media containing G418 $(0.5 \mathrm{mg} / \mathrm{ml})$. Individual clones that survived 2 weeks were cultured in positive selection medium for 8 weeks. Expression of HA-OX2R, HA-M1, HA-M2, and HA-M3 was assessed by western blotting using an anti-HA antibody (dilution, 1:1,000), as described below. For some experiments, transient transfection was performed (as described below).

Enzyme-linked immunosorbent assay (ELISA) to assess total and surface expression of mutants. Cells stably expressing HA-OX2R, HA-M1, HA-M2, and HA-M3 were plated into 96-well plates at a density of $10^{4}$ cells/well and incubated for $24 \mathrm{~h}$. Cells were then fixed in $4 \%$ paraformaldehyde prepared in phosphate-buffered saline (PBS) at $37^{\circ} \mathrm{C}$ for $1 \mathrm{~h}$, with or without $0.25 \%$ Triton X-100 for total HA or surface HA expression analysis, respectively. Cells were subsequently washed three times with PBS, and nonspecific binding sites were blocked with blocking buffer (3\% dry milk) at room temperature for $1 \mathrm{~h}$. Cells were incubated with a primary polyclonal anti-HA antibody (dilution, 1:500) overnight at $4^{\circ} \mathrm{C}$, and then washed three times with PBS. The following day, cells were incubated with a horseradish peroxidase-conjugated goat anti-rabbit immuniglobulin (Ig) G secondary antibody (dilution, $1: 2,000$ ) for $1 \mathrm{~h}$ at $37^{\circ} \mathrm{C}$, and then washed three times with PBS. 3,3',5,5'-tetramethylbenzidine substrate (200 $\mu 1$; Sigma-Aldrich; Merck Millipore) was added and cells were incubated for $30 \mathrm{~min}$ at $37^{\circ} \mathrm{C}$. The enzymatic reaction was stopped with $2 \mathrm{~N} \mathrm{H}_{2} \mathrm{SO}_{4}$ solution. Finally, each well was measured using an iMark ${ }^{\mathrm{TM}}$ Microplate Absorbance Reader (Bio-Rad Laboratories, Inc., Hercules, CA, USA) at a wavelength of $450 \mathrm{~nm}$.

cAMP production assay. Stably transfected cells were cultured in 12-well plates at a density of $5 \times 10^{5}$ cells/well and preincubated in stimulation buffer consisting of Dulbecco's modified Eagle's medium with $500 \mu \mathrm{M} \mathrm{IBMX}$ and $10 \mathrm{mM} \mathrm{MgCl}_{2}$, at $37^{\circ} \mathrm{C}$ for $20 \mathrm{~min}$. Cells were then stimulated with buffer containing either orexin-A (0.1-1,000 nM), orexin-B (0.1-1,000 nM) for $10 \mathrm{~min}$ at $37^{\circ} \mathrm{C}$. cAMP levels were determined using a Cyclic AMP assay kit (Cell Signaling Technology, Inc.) according to the manufacturer's instructions. Optical density at $450 \mathrm{~nm}$ was measured using a microplate reader.

Inositol phosphate (IP) accumulation assay. Stably transfected cells were plated in 24-well plates at a density of $2 \times 10^{5}$ cells/well. The following day, cells were treated with orexin-A $(100 \mathrm{nM})$ or orexin-B $(100 \mathrm{nM})$ prepared in stimulation buffer containing $10 \mathrm{mM}$ HEPES, $1 \mathrm{mM} \mathrm{CaCl}_{2}, 4.2 \mathrm{mM}$ $\mathrm{KCl}, 0.5 \mathrm{mM} \mathrm{MgCl}_{2}, 146 \mathrm{mM} \mathrm{NaCl}, 50 \mathrm{mM} \mathrm{LiCl}$ and $5.5 \mathrm{mM}$

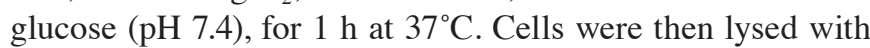
$2.5 \%$ lysis reagent (Beyotime Institute of Biotechnology, 
Shanghai, China) in $5 \% \mathrm{CO}_{2}$ at $37^{\circ} \mathrm{C}$ for $30 \mathrm{~min}$, according to the manufacturer's protocol. IP levels were measured using a Human Inositol Triphosphate ELISA kit (cat no. MBS024296; MyBioSource, San Diego, CA, USA), according to the manufacturer's protocol. The optical density at $490 \mathrm{~nm}$ was measured using a microplate reader.

Calcium release. Calcium signals in cells were detected using the Fluo-4 NW Calcium assay kit (Invitrogen; Thermo Fisher Scientific, Inc.) according to the manufacturer's instructions. Stably transfected cells at a density of $10^{4}$ cells/well were cultured in a black poly-D-lysine-coated 96-well plate for $24 \mathrm{~h}$. The following day, cells were stimulated for 5 min with $100 \mathrm{nM}$ orexin-A or orexin-B, washed twice with assay buffer, and $100 \mu 1$ loading dye solution was added to each well. Cells were subsequently incubated at $37^{\circ} \mathrm{C}$ for $30 \mathrm{~min}$, and then at room temperature for an additional $30 \mathrm{~min}$. The plates were washed three times with assay buffer. Fluorescence was measured using a TriStar LB 941 dual luciferase reporter-ready microplate reader (Berthold Technologies GmbH, Bad Wildbad, Germany), at an excitation wavelength of $485 \mathrm{~nm}$ and emission wavelength of $525 \mathrm{~nm}$.

Internalization of OX2R and truncation mutants. HEK293 cells were plated at a density of $2 \times 10^{4}$ cells in 96 -well plates. Cells transiently transfected with HA-OX2R, HA-M1, HA-M2, or HA-M3 was co-transfected with pcDNA3.1- $\beta$-arrestin1 or pcDNA3.1- $\beta$-arrestin2 using Lipofectamine 2000 according to the manufacturer's instructions (Invitrogen; Thermo Fisher Scientific, Inc.). pcDNA3.1- $\beta$-arrestin1/ $\beta$-arrestin2 recombinant plasmids were kindly provided by Professor Karin Eidne (QEII Medical Centre, Nedlands, Australia). Following further incubation with $100 \mathrm{nM}$ orexin-A or orexin-B for 10 , 30 and $60 \mathrm{~min}$, cells were fixed for $30 \mathrm{~min}$ at $37^{\circ} \mathrm{C}$ using $4 \%$ paraformaldehyde prepared in PBS. Cells were then washed with PBS and blocked at room temperature for $1 \mathrm{~h}$ using $3 \%$ dry milk. Cells were subsequently incubated with a primary polyclonal anti-HA antibody (dilution, 1:500) overnight at $4^{\circ} \mathrm{C}$. The following day, cells were washed three times with PBS, and incubated with a horseradish peroxidase-conjugated goat anti-rabbit IgG secondary antibody (dilution, 1:2,000) for $1 \mathrm{~h}$ at room temperaure. Cells were then washed and incubated with the 3,3',5,5'-tetramethylbenzidine substrate (Sigma-Aldrich; Merck Millipore) for $30 \mathrm{~min}$. Finally, the optical density was measured at a wavelength of $450 \mathrm{~nm}$ using a microplate reader.

Western blotting. Stably transfected cells at a density of $1 \times 10^{5}$ cells/well were cultured in 6 -well plates until they reached $90 \%$ confluence. Following serum starvation for $3 \mathrm{~h}$, cells were stimulated with $100 \mathrm{nM}$ orexin-A or $100 \mathrm{nM}$ orexin-B for $10 \mathrm{~min}$, immediately washed with ice-cold $\mathrm{PBS}$, and lysed in radioimmunoprecipitation assay lysis buffer for $30 \mathrm{~min}$ at $4^{\circ} \mathrm{C}$. The supernatants were collected by centrifugation at $13,400 \mathrm{x}$ a at $4^{\circ} \mathrm{C}$ for $30 \mathrm{~min}$, and the protein concentration was determined using a bicinchoninic assay kit (Nanjing KeyGen Biotech Co., Ltd., Nanjing, China). Equal amounts of extracted protein samples $(50 \mathrm{mg}$ ) were separated by $10 \%$ SDS-PAGE and transferred onto a polyvinylidene difluoride membrane. The membrane was blocked with $5 \%$ skimmed milk at room temperature for $1 \mathrm{~h}$ and incubated with a primary anti-p-ERK1/2 (Thr202/Tyr204) antibody (Cell Signaling Technology, Inc.; dilution, $1: 1,000)$ at $4^{\circ} \mathrm{C}$ overnight. The following day, the membrane was washed three times with TBS containing $0.1 \%$ Tween-20 (TBST), and then incubated with a horseradish peroxidase-conjugated anti-rabbit secondary antibody (dilution, 1:2,000) for $1 \mathrm{~h}$ at room temperature. The membrane was subsequently washed three times in TBST and signals were detected by enhanced chemiluminescence (ECL) using an ECL detection reagent (Beyotime Institute of Biotechnology). As a loading control, the membranes were stripped of the anti-p-ERK1/2 antibody using stripping buffer (Beijing Solarbio Science \& Technology Co., Ltd., Beijing, China; SW3020), and labeled with an anti-total (t)ERK1/2 antibody (Cell Signaling Technology, Inc.; dilution, 1:1,000) at $4^{\circ} \mathrm{C}$ overnight. Finally, the gray value of bands was calculated using ImageJ software version 2.1.4.6 (National Institutes of Health, Bethesda, MD, USA). The results are expressed as the p-ERK/t-ERK ratio. $\beta$-actin was used as the loading control.

Statistical analysis. Quantitative data are presented as the mean \pm standard error of the mean. GraphPad Prism software (version 5.0; GraphPad Software, Inc., La Jolla, CA, USA) was used to analyze the results with multiple group comparisons. One-way analysis of variance followed by a Tukey's post hoc test was used for multiple group comparisons, and $\mathrm{P}<0.05$ was considered to indicate a statistically significant difference. All experiments were repeated at least three times, and representative experiments are shown.

\section{Results}

The C-terminus of $O X 2 R$ is important for its cell surface expression. The $\mathrm{C}$-terminus has been repeatedly demonstrated to be an indispensable region of GPCRs that govern their cell surface expression (6). In the present study, the effect of C-terminal truncation on the surface expression of OX2R was assessed using an ELISA. The cell surface expression of the three truncated receptors was significantly lower when compared with that of the wild-type receptor (Fig. 1B). The HA-M3 mutant, containing a 30-aa deletion at position 415-444 of the C-terminus, exhibited an $18.66 \%$ reduction in cell surface expression (Fig. 1B). Cell surface expression of the HA-M2 mutant, containing a 60 -aa deletion at position 385-444, and the HA-M1 mutant, containing a 76-aa deletion at position 369-444, were decreased by $29.51 \%$ and $28.73 \%$, respectively (Fig. 1B). Expression of HA-M2 was lower than that of HA-M3, implying that the 60 residues at position 385-444 demonstrate a remarkable cumulative effect on the surface expression of the OX2R receptor. No significant difference in expression was observed between HA-M2 and HA-M1 mutants, which indicates that the aa residues at position 369-384 demonstrate no obvious effect on OX2R surface expression.

$C$-terminus of $O X 2 R$ significantly affects downstream effectors. The production of cAMP was detected following stimulation of cells stably expressing OX2R and the three truncated receptors with various concentrations of orexin-A (0.1-1,000 nM) or orexin-B (0.1-1,000 nM). cAMP production was not significantly altered among orexin-A or 


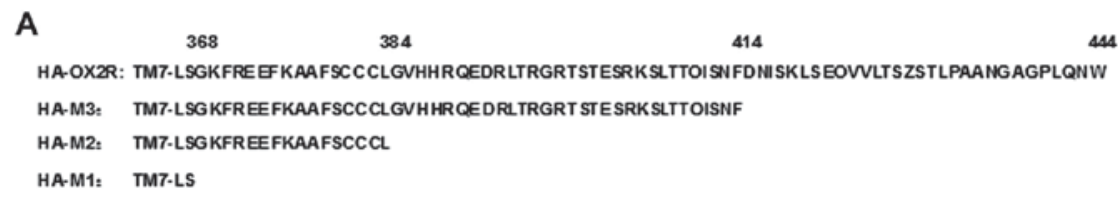

B

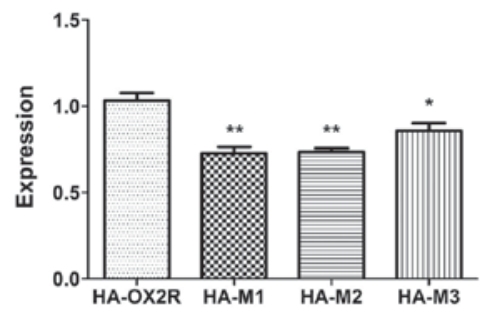

Figure 1. Schematic representation and cell surface expression of OX2R and $3 \mathrm{C}$-terminal truncation mutants. (A) The amino acid sequence and residue numbers of the OX2R C-terminus downstream of TM7 is shown. OX2R and the three truncation mutants were tagged with the HA epitope at the N-terminus. (B) Progressive truncation of the C-terminus decreased OX2R cell surface expression. ${ }^{*} \mathrm{P}<0.05$ and ${ }^{* *} \mathrm{P}<0.01$ vs. HA-OX2R. OX2R, orexin 2 receptor; TM7, transmembrane domain 7; HA, hemagglutinin.

orexin-B-treated cells transfected with the truncated receptors and cells transfected with wild-type OX2R. The half maximal effective concentration $\left(\mathrm{EC}_{50}\right)$ values for orexin A-treated cells were as follows: HA-M1, $294.1 \mathrm{nM}$; HA-M2, 142.9 nM; HA-M3, $281.6 \mathrm{nM}$ (Fig. 2A). The $\mathrm{EC}_{50}$ values for orexin-B-treated cells were as follows: HA-M1, $202.2 \mathrm{nM}$; HA-M2, $302.5 \mathrm{nM}$; and HA-M3, $352.2 \mathrm{nM}$ (Fig. 2B). These results indicated that the 76-aa in the 369-444 C-terminal region demonstrated no significant influence on cAMP production.

Following stimulation with orexin-A and orexin-B, $\mathrm{Ca}^{2+}$ release was not significantly altered among cells expressing HA-M3 and those expressing full-length HA-OX2R, which demonstrated that the 30 aa sequence at position 415-444 was not responsible for affecting $\mathrm{Ca}^{2+}$ release (Fig. 2C and D). Nevertheless, $\mathrm{Ca}^{2+}$ release was markedly lower in cells expressing HA-M1 and HA-M2 when compared with cells expressing $\mathrm{HA}-\mathrm{OX} 2 \mathrm{R}$. The $\mathrm{EC}_{50}$ values for orexin-A-treated cells were as follows: HA-M1, $255.2 \mathrm{nM}$; HA-M2, $250.5 \mathrm{nM}$; HA-M3, $123.9 \mathrm{nM}$ (Fig. 2C). The $\mathrm{EC}_{50}$ values for orexin-B-treated cells were as follows: HA-M1, $253.9 \mathrm{nM}$; HA-M2, 233.5 nM; $147.1 \mathrm{nM}$ for HA-M3 (Fig. 2D). These results indicated that the 30-aa sequence at position 385-414, which was deleted in HA-M2, and the 16-aa sequence at position 369-384, which was deleted in HA-M1, (residues 369-414) significantly influenced $\mathrm{Ca}^{2+}$ release.

To assess the effect of OX2R C-terminal truncation on $\mathrm{G}_{\mathrm{q}}$ subunit protein coupling, the abilities of OX2R and the three truncated receptors in stimulating IP production were examined. Following stimulation with $100 \mathrm{nM}$ orexin-A or $100 \mathrm{nM}$ orexin-B, IP production was significantly higher in cells expressing HA-M3 and HA-M2 when compared with their respective non-simulated controls (Fig. 2E). This indicated that the 30-aa sequence at position 415-444, which was deleted in HA-M3, and the 30-aa sequence at position 385-414, which was deleted in HA-M2, significantly influenced IP production. By contrast, IP production in cells expressing HA-M1 was not significantly altered between orexin-A or orexin-B-simulated and non-simulated cells (Fig. 2E), indicating that the 16-aa sequence at position 369-384, did not influence IP production.
C-terminus of $O X 2 R$ is important for receptor internalization. $\beta$-arrestin family members mediate desensitization of many GPCRs by uncoupling the stimulated receptors from their cognate G-proteins $(18,19)$. To assess whether OX2R and the C-terminal truncation mutants differ in their ability to recruit arrestin proteins, their physical associations with $\beta$-arrestin1 and $\beta$-arrestin 2 were tested using an ELISA. Following co-transfection with $\beta$-arrestin1, internalization of HA-M3 after stimulation with orexin-A or orexin-B, was not significantly affected at all time points examined (Fig. 3A and $\mathrm{B}$ ), which indicated that the 30 -aa sequence at position 415-444 was not important for receptor internalization. However, internalization of HA-M2 following stimulation with orexin-A or orexin-B following transfection with $\beta$-arrestin1, which lacked an additional 30-aa sequence at the 385-414 position, was significantly affected between 0 and 60 min compared with the full-length OX2R (Fig. 3). This suggests that the 30-aa sequence may be important for receptor internalization. Internalization of HA-M1 and HA-M2 in $\beta$-arrestin2-transfected cells following treatment with orexin-A or -B were not significantly different with each other, which suggests that residues 369-384 of the OX2R C-terminal domain may not be important for receptor internalization (Fig. 3). Similarly, this effect was observed following co-transfection with $\beta$-arrestin 2 and stimulation with orexin-A (Fig. 3C) or orexin-B (Fig. 3D). Therefore, the results suggest that the 385-414 region is the major site for physical association with arrestin.

C-terminus of OX $2 R$ affects ERK1/2 phosphorylation. In order to determine the effect of the $C$-terminal region of OX2R on ERK1/2 phosphorylation, the protein expression levels of p-ERK were determined following transfection of cells with the three $\mathrm{C}$-terminal truncation mutations and a full-length OX2R C-terminal sequence. Stably transfected cells were treated with $100 \mathrm{nM}$ orexin-A or orexin-B for $10 \mathrm{~min}$ and the level of ERK1/2 phosphorylation was detected. As shown in Fig. 4, ERK1/2 phosphorylation was significantly lower in orexin-A and orexin-B-treated cells stably transfected 
A
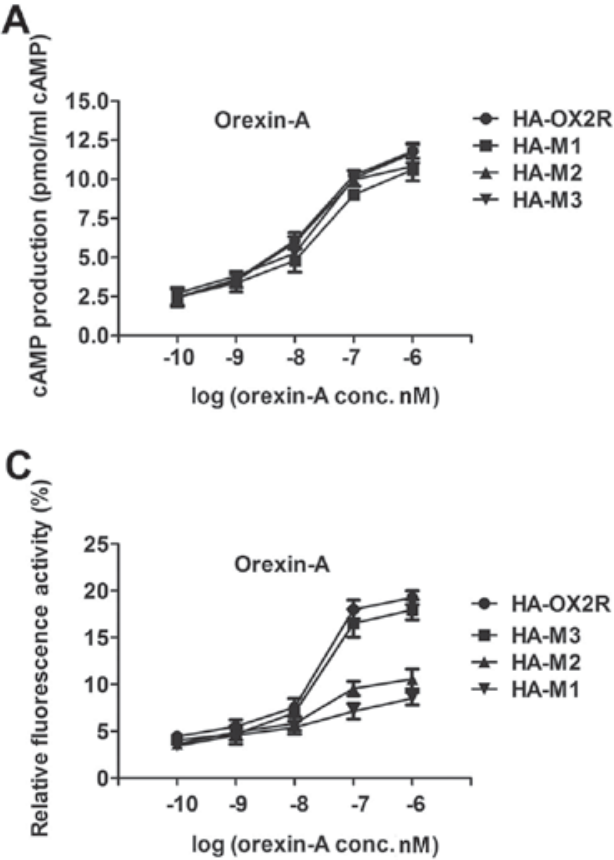

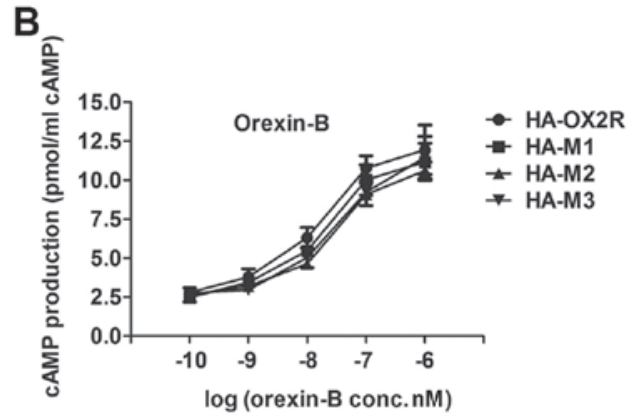

D

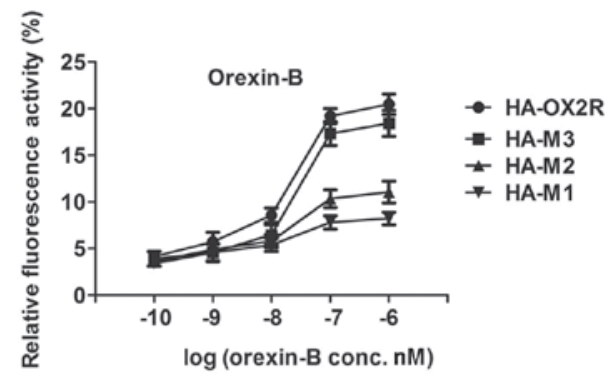

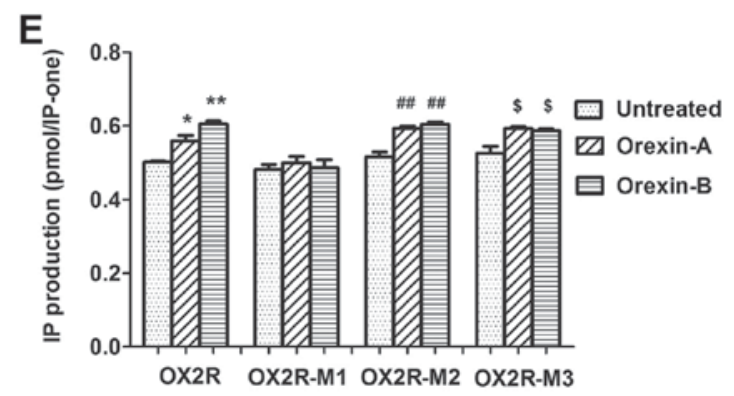

Figure 2. Influence of the full-length OX2R C-terminal region and three truncation mutants on downstream effectors. cAMP production was not significantly altered between cells transfected with the three truncation mutants and cells transfected with HA-OX2R following stimulation with (A) orexin-A or (B) orexin-B. $\mathrm{Ca}^{2+}$ release in cells transfected with full-length HA-OX2R or the three truncation mutants following stimulation with (C) orexin-A or (D) orexin-B. $\mathrm{Ca}^{2+}$ release was not significantly altered between cells expressing HA-M3 and cells expressing HA-OX2R following stimulation with orexin-A or orexin-B. By contrast, $\mathrm{Ca}^{2+}$ release was lower in cells expressing HA-M1 and HA-M2 when compared with cells expressing HA-OX2R. (E) A significant difference in IP production was observed between orexin-A (100 nM) or orexin-B (100 nM)-stimulated cells and non-simulated cells expressing HA-M3 and HA-M2, whereas, no significant difference between stimulated cells and non-simulated cells expressing HA-M1 was observed. ${ }^{*} \mathrm{P}<0.05$ and ${ }^{* *} \mathrm{P}<0.01$ vs. untreated cells transfected with HA-OX2R; ${ }^{\# \prime} \mathrm{P}<0.01$ vs. untreated cells transfected with OXR-M2; ${ }^{~} \mathrm{P}<0.05$ vs. untreated cells transfected with $\mathrm{OX} 2 \mathrm{R}-\mathrm{M} 3$. OX2R, orexin 2 receptor; cAMP, cyclic adenosine monophosphate; HA, hemagglutinin.

with HA-M1 when compared with the full-length sequence. By contrast, ERK1/2 phosphorylation was not significantly different in cells stably transfected with HA-M2 and HA-M3 when compared with those transfected with the full-length sequence (Fig. 4). The results indicate that the 16-aa sequence at position 369-384 in OX2R may influence ERK1/2 phosphorylation and potentially the downstream signaling pathway.

\section{Discussion}

The C-terminus is known to be involved in mediating the cell surface expression of GPCRs. For instance, serial truncation of the $\mathrm{C}-\mathrm{C}$ chemokine receptor type 5 may result in progressive loss of its cell surface expression (20). Tetsuka et al (21) demonstrated that C-terminal mutants of melanin-concentrating hormone receptor 1 (MCH1R) exhibit progressively reduced expression levels when compared with the wild-type protein. When progressive truncations were introduced at the
C-terminus of the gonadotropin-releasing hormone receptor (GnRH-R), the stop331 and stop337 mutants exhibited $40 \%$ cell surface expression when compared with wild-type GnRH-R (22). Alanine residues at the C-terminus are necessary for the cell surface expression of the glucagon-like peptide-2 receptor (23). In order to investigate the effect of the C-terminus on the cell surface expression of OX2R in the present study, three truncation mutants of OX2R were constructed. The three mutant receptors lacking aa sequences at the $\mathrm{C}$-terminus exhibited significantly reduced expression at the cell surface. Cell surface expression was not significantly altered between HA-M2 and HA-M1 mutants, which indicated that the aa sequence at position 369-384 demonstrated no obvious effect on the surface expression of this receptor. Therefore, aa sequences in the 385-444 region of the OX2R C-terminus are a key determinant for the localization or stability of the receptor at the cell surface. This domain must therefore contain sites essential for cell surface expression, and 
A

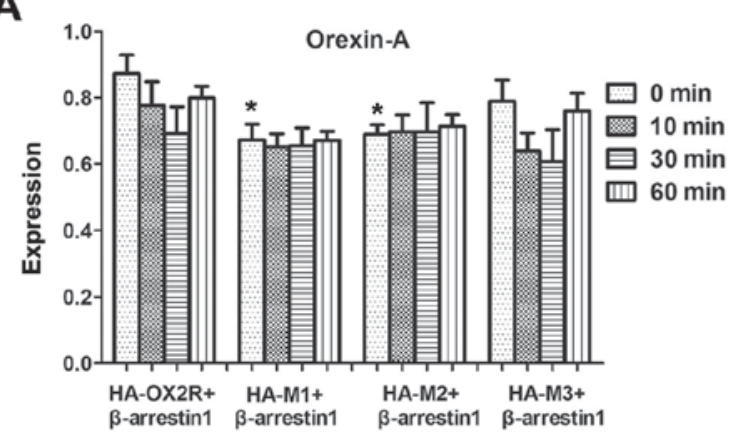

C

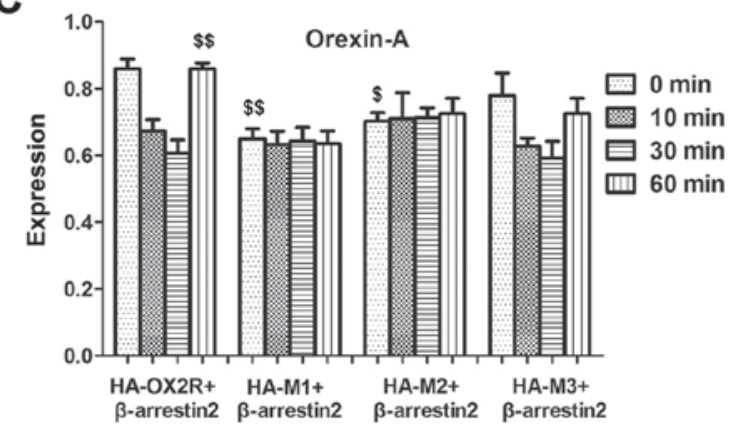

B

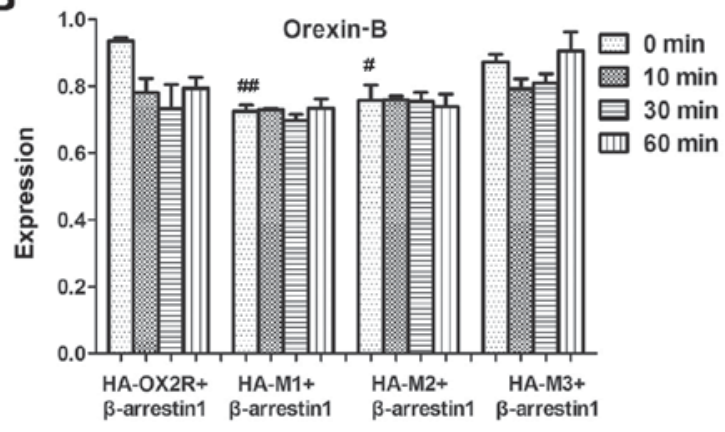

D

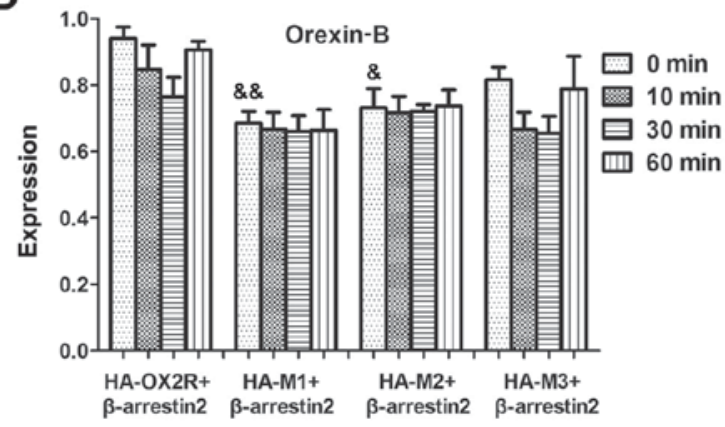

Figure 3. Internalization of OX2R following transfection of the full-length C-terminal region and 3 truncation mutants. Following co-transfection with $\beta$-arrestin1 and stimulation with (A) orexin-A $(100 \mathrm{nM})$ or $(B)$ orexin-B $(100 \mathrm{nM})$, internalization of HA-M1 and HA-M2 was significantly affected compared with HA-OX2R, whereas the internalization of HA-M3 was not affected compared with HA-OX2R. Following co-transfection with $\beta$-arrestin2 and stimulation with $(\mathrm{C})$ orexin-A $(100 \mathrm{nM})$ or $(\mathrm{D})$ orexin-B $(100 \mathrm{nM})$, internalization of HA-M1 and HA-M2 was significantly altered when compared with HA-OX2R-transfected cells, whereas no significant alteration in HA-M3 internalization was observed. ${ }^{*} \mathrm{P}<0.05$ vs. orexin-A+HA-OX2R+beta-arrestin1 at $0 \mathrm{~min} ;{ }^{~} \mathrm{P}<0.05,{ }^{\# \#} \mathrm{P}<0.01$ vs. orexin-B+HA-OX2R+beta-arrestin1 at $0 \mathrm{~min} ;{ }^{\$} \mathrm{P}<0.05,{ }^{\$ \$} \mathrm{P}<0.01$ vs. orexin- $\mathrm{A}+\mathrm{HA}-\mathrm{OX} 2 \mathrm{R}+$ beta-arrestin 2 at 0 min; ${ }^{\circledR} \mathrm{P}<0.05$, \&\& $\mathrm{P}<0.01$ vs. orexin-B+HA-OX2R+beta-arrestin 2 at 0 min. OX2R, orexin 2 receptor; HA, hemagglutinin.
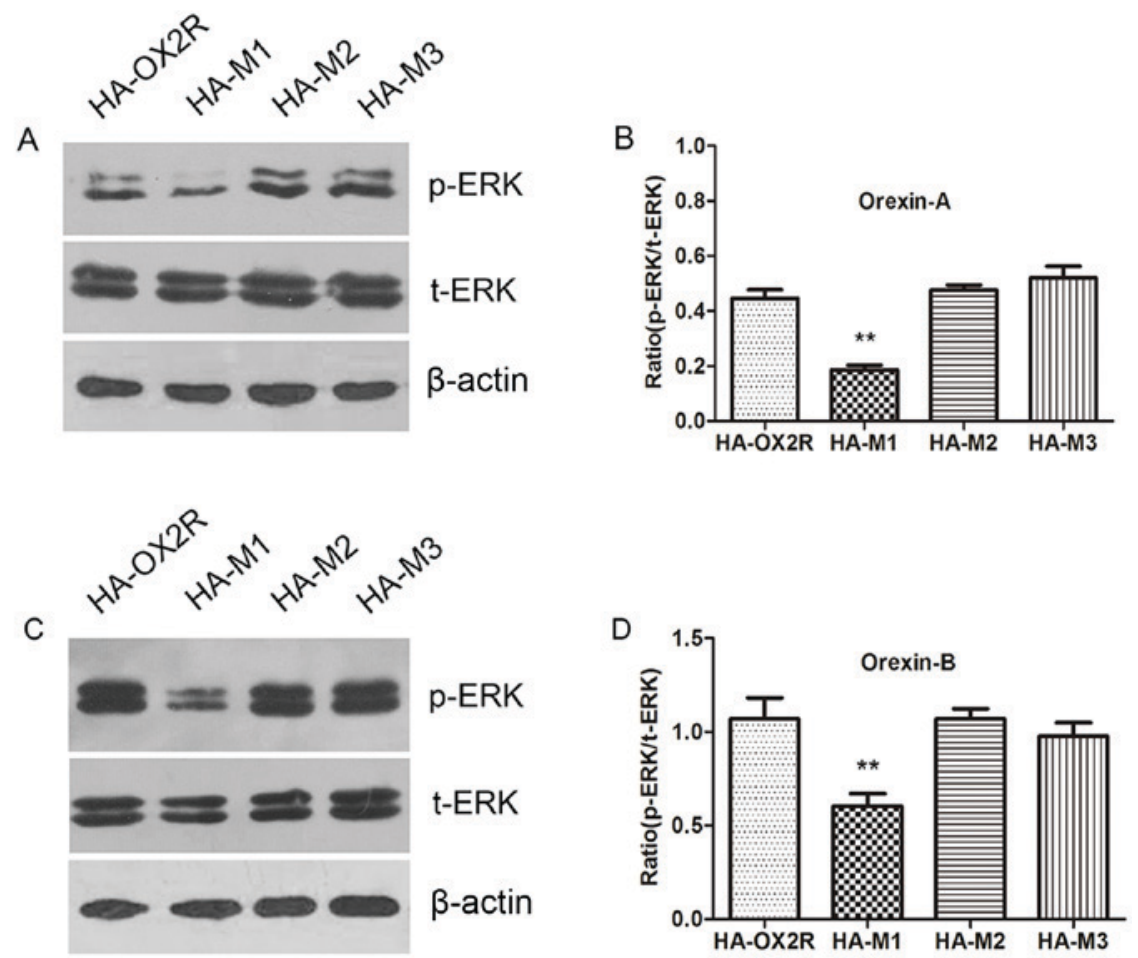

Figure 4. Expression of p-ERK1/2 in cells expressing OX2R and 3 truncation mutants. (A) Western blot analysis of p-ERK1/2 expression and (B) quantification of band intensities in HA-OX2R, HA-M1, HA-M2 and HA-M3-transfected cells following stimulation with $100 \mathrm{nM}$ orexin-A for $10 \mathrm{~min}$. (C) Western blot analysis of p-ERK1/2 expression and (D) quantification of band intensities in HA-OX2R, HA-M1, HA-M2 and HA-M3-transfected cells following stimulation with $100 \mathrm{nM}$ orexin-B. ERK1/2 phosphorylation was significantly lower in cells expressing HA-M1 when compared with HA-OX2R-transfected cells. However, no significant difference in ERK1/2 phosphorylation was observed between cells expressing HA-M2, HA-M3 and HA-OX2R. ${ }^{* *} \mathrm{P}<0.01$ vs. HA-OX2R. ERK1/2, extracellular signal-regulated kinase 1/2; p-ERK, phosphorylated ERK; t-ERK, total-ERK; OX2R, orexin 2 receptor; HA, hemagglutinin. 
future studies will focus on identifying and mutating these sites in order to confirm their functional role.

Following activation of a GPCR, several dispersed loci facilitate internalization. Phosphorylation of aa sequences at the $\mathrm{C}$-terminus is required for interaction with $\beta$-arrestin, leading to receptor internalization. The precise molecular nature of GPCR internalization is complicated and involves numerous protein partners $(24,25)$. Using a series of truncation and deletion mutants, Chaki et al (26) demonstrated that the 310-327 region is required for internalization of the rat angiotensin II type $1 \mathrm{~A}$ receptor. The non-mammalian GnRH-R shows rapid desensitization (22) and agonist-induced internalization (27) when aa sequences are phosphorylated in its C-terminal tail. The C-terminus of the human prostaglandin E2 receptor subtype EP4 contains 38 Ser/Thr phosphorylation sites. These residues are necessary for $\beta$-arrestin 1 recruitment and agonist-induced internalization (28). The results of the present study demonstrated that the aa sequences at position 385-414 of the OX2R C-terminal domain may be the major site for agonist-induced internalization. This domain contains ten potential Ser/Thr phosphorylation sites important for coupling to $\beta$-arrestin, leading to internalization $(29,30)$. The results of the current study are consistent with those presented by Golan et al (25), which demonstrated that putative Ser/Thr clusters in the C-terminus of OX2R are involved in receptor- $\beta$-arrestin-ubiquitin complex formation.

The signal transduction of GPCRs is primarily mediated by the third intracellular loop and the C-terminal sequence (31). Over the past few years, a number of studies have increasingly highlighted the importance of the C-terminal sequence for intracellular signal transduction. Lehmann et al (19) analyzed two C-terminal mutants of MCH1R, one of which contained a 36-aa deletion, while the other possessed a 32-aa deletion. The sequence between Phe318 and Arg321 was observed to be responsible for signal transduction. In addition, mutation of either Arg319 or Lys320, but not Arg321, significantly reduced $\mathrm{Ca}^{2+}$ influx (21). For the glucagon-like peptide-2 receptor, the major C-terminal sequence is dispensable for cAMP accumulation, ERK1/2 activation and endocytosis (23). Tang et al (17) reported that OX2R may activate the ERK1/2 signaling pathway via $G_{q}, G_{s}$, and $G_{i}$ subunits; with $G_{q}$ being the major mediator. In the present study, the OX2R truncation mutations demonstrated no significant effects on cAMP production. This result is consistent with the finding that the $\mathrm{C}$-terminal tail of one family B GPCR is not required for coupling to the $\mathrm{G}_{\mathrm{s}}$ subunit (32). However, the OX2R truncation mutations demonstrated a marked effect on IP production and $\mathrm{Ca}^{2+}$ release in the current study. This implied that the $\mathrm{C}$-terminus of OX2R may be indispensable for the $G_{q}$ signaling pathway. In addition, residues 369-384 at the $\mathrm{C}$-terminus influenced the expression of p-ERK1/2. Willars et al (27) demonstrated that orexin A (100 nM) increased the labeling of OX2R with $\mathrm{G}_{\mathrm{s}}$ and $G_{I}$ subunits, however the same effect was not observed for $G_{o}$ and $G_{q}$ subunits. In addition, the authors indicated that OX2R binds to $G_{s}$ and $G_{i}$ subunits in the human fetal adrenal cortex, whereas a shift towards $G_{q}$ and less towards $G_{i}$ occurs in the adult adrenal gland (28). Therefore, the authors of the present study hypothesized that the coupling of orexin receptors to multiple $\mathrm{G}$ proteins may be diverse in different cell lines and under different stimulus conditions.
The present study demonstrated that aa residues in the $\mathrm{C}$-terminus serve an important role in the expression and signal transduction of OX2R. In addition, the aa residues in different locations possess evidentially different roles. The aa residues in the C-terminus of OX2R were analyzed using bioinformatics. A series of conserved residues were identified, which reportedly serve an indispensable role in receptor expression and the downstream signaling pathways (unpublished data). In future studies, C-terminal mutants will be constructed whereby one or several of the conserved residues are mutated, in order to determine the effects of these mutations on the expression and signal transduction of the OX2R receptor.

\section{Acknowledgements}

The present work was supported by grants from the National Nature Science Foundation of China (grant nos. 81501018 and 31271243) and the Shandong Province Natural Science Foundation (grant no. ZR2013CQ031) and by a starting grant for Doctor of Jining Medical University.

\section{References}

1. Bockaert J and Pin JP: Molecular tinkering of G protein-coupled receptors: An evolutionary success. Embo J 18: 1723-1729, 1999.

2. Croft W, Hill C, McCann E, Bond M, Esparza-Franco M, Bennett J, Rand D, Davey J and Ladds G: A physiologically required $\mathrm{G}$ protein-coupled receptor (GPCR)-regulator of $\mathrm{G}$ protein signaling (RGS) interaction that compartmentalizes RGS activity. J Biol Chem 288: 27327-27342, 2013.

3. Lu ZL, Saldanha JW and Hulme EC: Seven-transmembrane receptors: Crystals clarify. Trends Pharmacol Sci 23: 140-146, 2002.

4. Wistrand M, Käll L and Sonnhammer EL: A general model of G protein-coupled receptor sequences and its application to detect remote homologs. Protein Sci 15: 509-521, 2006.

5. Fortin JP, Zhu Y, Choi C, Beinborn M, Nitabach MN and Kopin AS: Membrane-tethered ligands are effective probes for exploring class B1 G protein-coupled receptor function. Proc Natl Acad Sci USA 106: 8049-8054, 2009.

6. Thompson A and Kanamarlapudi V: Distinct regions in the C-Terminus required for GLP-1R cell surface expression, activity and internalisation. Mol Cell Endocrinol 413: 66-77, 2015.

7. Feng Y, Liu T, Li XQ, Liu Y, Zhu XY, Jankovic J, Pan TH and Wu YC: Neuroprotection by Orexin-A via HIF-1 $\alpha$ induction in a cellular model of Parkinson's disease. Neurosci Lett 579: 35-40, 2014.

8. Lowther KM, Uliasz TF, Götz KR, Nikolaev VO and Mehlmann LM: Regulation of constitutive GPR3 signaling and surface localization by GRK2 and $\beta$-arrestin-2 Overexpression in HEK293 cells. PLoS One 8: e65365, 2013.

9. Katsushima Y, Sato T, Yamada C, Ito M, Suzuki Y, Ogawa E, Sukegawa I, Sukegawa J, Fukunaga $\mathrm{K}$ and Yanagisawa T: Interaction of PICK1 with C-terminus of growth hormonereleasing hormone receptor (GHRHR) modulates trafficking and signal transduction of human GHRHR. J Pharmacol Sci 122: 193-204, 2013.

10. Shim JY, Ahn KH and Kendall DA: Molecular basis of cannabinoid $\mathrm{CB} 1$ receptor coupling to the $\mathrm{G}$ protein heterotrimer Gaiß $\gamma$ : identification of key CB1 contacts with the C-terminal helix $\alpha 5$ of Gai. J Biol Chem 288: 32449-32465, 2013.

11. Gandía J, Fernández-Dueñas V, Morató X, Caltabiano G, González-Muñiz R, Pardo L, Stagljar I and Ciruela F: The Parkinson's disease-associated GPR 37 receptor-mediated cytotoxicity is controlled by its intracellular cysteine-rich domain. J Neurochem 125: 362-372, 2013.

12. Ammoun S, Holmqvist T, Shariatmadari R, Oonk HB, Detheux M, Parmentier M, Akerman KE and Kukkonen JP: Distinct recognition of OX1 and OX2 receptors by orexin peptides. J Pharmacol Exp Ther 305: 507-514, 2003.

13. Baccari MC: Orexins and gastrointestinal functions. Curr Protein Pept Sci 11: 148-155, 2010. 
14. Mavanji V,Perez-Leighton CE, Kotz CM,Kotz CM,Billington CJ, Parthasarathy S, Sinton CM and Teske JA: Promotion of wakefulness and energy expenditure by Orexin-A in the ventrolateral preoptic area. Sleep 38: 1361-1370, 2015.

15. Kannan H, Shirasaka T, Watanabe S, Yu NS, Kuitake T and Takasaki M: Central action of orexins on sympathetic outflow and cardiovascular function with a focus on the paraventricular nucleus of the hypothalamus. Masui 56: 30-39, 2007 (In Japanese).

16. Kaminski T and Smolinska N: Expression of orexin receptors in the pituitary. Vitam Horm 89: 61-73, 2012.

17. Tang J, Chen J, Ramanjaneya M, Punn A, Conner AC and Randeva HS: The signalling profile of recombinant human orexin-2 receptor. Cell Signal 20: 1651-1661, 2008.

18. Kara E, Crépieux P, Gauthier C, Martinat N, Piketty V, Guillou F and Reiter E: A phosphorylation cluster of five serine and threonine residues in the $\mathrm{C}$-terminus of the follicle-stimulating hormone receptor is important for desensitization but not for beta-arrestin-mediated ERK activation. Mol Endocrinol 20 : 3014-3026, 2006.

19. Lehmann A, Kliewer A, Schutz D, Nagel F, Stumm R and Schulz S: Carboxyl-terminal multi-site phosphorylation regulates internalization and desensitization of the human sst2 somatostatin receptor. Mol Cell Endocrinol 387: 44-51, 2014

20. Venkatesan S, Petrovic A, Locati M, Kim YO, Weissman D and Murphy PM: A membrane-proximal basic domain and cysteine cluster in the C-terminal tail of CCR5 constitute a bipartite motif critical for cell surface expression. J Biol Chem 276: 40133-40145, 2001.

21. Tetsuka M, Saito Y, Imai K, Doi H and Maruyama K: The basic residues in the membrane-proximal $\mathrm{C}$-terminal tail of the rat melanin-concentrating hormone receptor 1 are required for receptor function. Endocrinology 145: 3712-3723, 2004.

22. Blomenröhr M, Heding A, Sellar R, Leurs R, Bogerd J, Eidne KA and Willars GB: Pivotal role for the cytoplasmic carboxyl-terminal tail of a nonmammalian gonadotropin-releasing hormone receptor in cell surface expression, ligand binding, and receptor phosphorylation and internalization. Mol Pharmacol 56: 1229-1237, 1999.

23. Estall JL, Koehler JA, Yusta B and Drucker DJ: The glucagon-like peptide- 2 receptor $\mathrm{C}$ terminus modulates beta-arrestin-2 association but is dispensable for ligand-induced desensitization, endocytosis, and G-protein-dependent effector activation. J Biol Chem 280: 22124-22134, 2005.
24. Innamorati G, Giannone F, Guzzi F, Rovati GE, Accomazzo MR, Chini B, Bianchi E, Schiaffino MV, Tridente G and Parenti M: Heterotrimeric $G$ proteins demonstrate differential sensitivity to beta-arrestin dependent desensitization. Cell Signal 21: 1135-1142, 2009.

25. Golan M, Schreiber G and Avissar S: Antidepressants, beta-arrestins and GRKs: From regulation of signal desensitization to intracellular multifunctional adaptor functions. Curr Pharm Des 15: 1699-1708, 2009.

26. Chaki S, Guo DF, Yamano Y, Ohyama K, Tani M, Mizukoshi M, Shirai $\mathrm{H}$ and Inagami T: Role of carboxyl tail of the rat angiotensin II type $1 \mathrm{~A}$ receptor in agonist-induced internalization of the receptor. Kidney Int 46: 1492-1495, 1994.

27. Willars GB, Heding A, Vrecl M, Sellar R, Blomenröhr M, Nahorski SR and Eidne KA: Lack of a C-terminal tail in the mammalian gonadotropin-releasing hormone receptor confers resistance to agonist-dependent phosphorylation and rapid desensitization. J Biol Chem 274: 30146-30153, 1999.

28. Neuschäfer-Rube F, Hermosilla R, Rehwald M, Rönnstrand L, Schülein R, Wernstedt $C$ and Püschel GP: Identification of a $\mathrm{Ser} / \mathrm{Thr}$ cluster in the C-terminal domain of the human prostaglandin receptor EP4 that is essential for agonist-induced beta-arrestin1 recruitment but differs from the apparent principal phosphorylation site. Biochem J 379: 573-585, 2004.

29. Qiu Y, Loh HH and Law PY: Phosphorylation of the delta-opioid receptor regulates its beta-arrestins selectivity and subsequent receptor internalization and adenylyl cyclase desensitization. J Biol Chem 282: 22315-22323, 2007.

30. Liu Q, Dewi DA, Liu W, Bee MS and Schonbrunn A: Distinct phosphorylation sites in the SST2A somatostatin receptor control internalization, desensitization, and arrestin binding. Mol Pharmacol 73: 292-304, 2008.

31. Conchon S, Barrault MB, Miserey S, Corvol P and Clauser E: The C-terminal third intracellular loop of the rat AT1A angiotensin receptor plays a key role in $\mathrm{G}$ protein coupling specificity and transduction of the mitogenic signal. J Biol Chem 272: 25566-25572, 1997.

32. Conner M, Hicks MR, Dafforn T, Knowles TJ, Ludwig C, Staddon S, Overduin M, Günther UL, Thome J and Wheatley M: Functional and biophysical analysis of the C-terminus of the CGRP-receptor; a family B GPCR. Biochemistry 47: 8434-8444, 2008. 